International Review of Sociology

Revue Internationale de Sociologie

\title{
The dimensions of gender in the last twenty years: an analysis of the International Review of Sociology
}

\section{Isabella Mingo \& Mariella Nocenzi}

To cite this article: Isabella Mingo \& Mariella Nocenzi (2020): The dimensions of gender in the last twenty years: an analysis of the International Review of Sociology, International Review of Sociology, DOI: $10.1080 / 03906701.2020 .1724368$

To link to this article: https://doi.org/10.1080/03906701.2020.1724368

\section{曲 Published online: 13 Feb 2020.}

Submit your article to this journal $\widetilde{x}$

Q View related articles $\sqsubset$

View Crossmark data $\asymp$ 


\title{
The dimensions of gender in the last twenty years: an analysis of the International Review of Sociology
}

\author{
Isabella Mingo (D) and Mariella Nocenzi \\ Department of Communication and Social Research, Sapienza University of Rome, Rome, Italy
}

\begin{abstract}
The Social Sciences and, specifically, the sociological research, have progressively assumed the gender factor as one of the strategic keys to understand contemporary phenomena. In fact, as a variable for socio-statistical analysis or as a characterizing trait of individual identity, it is a decisive factor in the interpretation of the deep social transformations, and it inspires the self-reflection of the sociologists about the analytical tools of their discipline. The contribution proposes, through a lexicometric approach, an analysis of the articles published in the last two decades by the oldest journal of Sociology, published by Routledge. The main aim is to highlight the different ways in which gender issues are declined in the international sociological researches presented in the repertoire of the International Review of Sociology and to outline, both on the lexical level and on the topic level, the changes occurred over time.
\end{abstract}

\section{ARTICLE HISTORY}

Received 15 October 2019

Accepted 13 January 2020

\section{KEYWORDS}

Gender; International Review of Sociology; lexicometric analysis; textual analysis; social change

\section{Introduction ${ }^{1}$}

From 1955, when in a relevant paper the American scholar Money, Hampson, and Hampson $(1955, \mathrm{ix})$ coined the term of gender for the definition of 'those things that a person says or does to disclose himself or herself as having the status of boy or man, girl or woman', the social sciences have developed entire subfields and a wide range of topics to analyse it with a variety of research methods.

Especially sociologists had outlined specific theoretical approaches and had done many detailed studies to understand firstly what gender is and the difference with sex. They had shared that if the meaning of sex is the biological classification based on body parts, gender, on the other hand, is the social classification based on one's identity, presentation of self, behavior, and interaction with others. Sociologists, hence, view gender as a learned behavior and a culturally produced identity, and, for these reasons, they define it as a 'social' category. It has always been a very relevant category for the critical analysis of the social construction because one of the most important social structures is the status and one of the most strategic statuses is just gender.

In the last decades, the sociological theories and researches based on gender are become more and more widespread, articulated, integrated with other subfields of sociology and of

CONTACT Mariella Nocenzi mariella.nocenzi@uniroma1.it $\Theta$ Department of Communication and Social Research, Sapienza University of Rome, Via Salaria 113 I-00198 Rome, Italy 
the other social sciences. One of the most representative indicators of this research development and specialization is not only the common recognition and, then, institution of the sociology of the gender as a subfield of the sociology, but the most frequent use of gender as reference concept for all the other sociological theoretical approaches to the analysis of the social processes. The same sociology of gender has studied many topics with multiple research methods, including identity, social interaction, power and oppression, and the interaction with race, class, culture, religion, and sexuality, among others.

This paper aims to observe and, if possible, to interpret this progressive diffusion and specialization in the use of gender as a theoretical and research category through the publications of the International Review of Sociology (IRS), a sociological journal, edited by Routledge with a worldwide online and paper diffusion, during the last two decades. This journal, the oldest review in the field of sociology in Europe, founded by René Worms in 1893 in Paris, still maintains - as the 'Aims and scope of the Review' state -

the traditional orientation of the journal as well as of the world's first international academic organization of sociology which started as an association of contributors to IRS: it assumes that sociology is not conceived apart from economics, history, demography, anthropology and social psychology. Rather, sociology is a science which aims to discover the links between the various areas of social activity and not just a set of empty formulas. Thus, International Review of Sociology provides a medium through which up-to-date results of interdisciplinary research can be spread across disciplines as well as across continents and cultures. ${ }^{2}$

The IRS scientific profile has been selected because it permits to refer to its production for the aims of this paper as an international representative sociological repertoire of the last decades. The main evidences of this research could be used as a reference to be tested thanks to further analysis of other international sociological reviews production.

The Authors propose to highlight the different ways in which gender issues are declined in the international sociological researches, through an analysis of the articles published in the last two decades (1997-2017) in IRS. We consider the last two decades of publication not only because of the best accessibility to the IRS catalogue. For the sociology, indeed, the recent gender studies and researches have registered a deeper specialization in terms of connection with other disciplines, unusual application of the gender approach to some social phenomena, exploration of new research frontiers (multiple gender identities, gender sensitive data arrangement, the non-alignment statuses of sex and gender et similia).

The paper will delve into these issues, first exploring the developing meaning of gender in the sociological analysis in the last decades through the main theoretical currents and research networks in the international scientific community, where the authors of the Review live and work.

The introduction of the gender studies and of their last transformations will be represented by a selected corpus of research articles edited by the Review from 1997 to 2017. The second part of the paper will describe the empirical part of this research, starting from the collection of data and the selection of the methodological tools. The criteria of the selection and the outcomes of the preliminary analysis of the corpus will be explained and, then, the selected strategies for this analysis: each of them - from the lexical analysis to the correspondence analysis and the cluster analysis - has given some important lexical outputs for a sociological analysis. 
In the third part, these outputs will contribute to highlight some of the current trends in the sociological theoretical and empirical research about gender, as they can be represented in those publications of the Review by important scholars of the international scientific community. The hypothesis of a growing systematic specialization in the gender studies, the new crossing fields of research that assume the gender as the main research factor and the signs of a possible 'gendered' research on the gender issues will be verified.

\section{The developing role of gender in the recent sociological research}

In the recent decades the prominent subfield of sociology, the sociology of gender, has become something more than the branch of a discipline that wants to interpret the social interactions based on the status of gender - one of the most important social structures correlated to the social relations.

If fourty years ago the social structures of gender and sex are used reversed (Saltzman Chafetz, 1978), in the last decades the general scientific consensus on the use of gender only linked to socio-cultural aspects reflects the changing individual identity in the contemporary social institutions - family, school, workplace, peer group etc. This change has been as innovative as the identification based on the gender between men and women from the Industrial Revolution: the distinction between men as breadwinners and women as housewives didn't characterize pre-industrial divisions of labor, while the social relations changed in the industrial society where separated roles in home and work took shape.

After this social change and sociological acquisition, the major sociological frameworks proposed a specific views and theoretical interpretations about gender, how it affect the other aspects of society, how it is interconnected to the other factors of change.

Thanks to their systematic vision of the society the functional theorists were the first sociologists to classify social roles and expectations in a gender-oriented scheme gathering for men instrumental roles and for women expressive roles in a perfect order for the benefit of the society. A modern and progressive society, as they tought, can hold only on a gendered division of labor and all the socialization stages in the life of the individual are to be articulated to encourage distinct - and not equal - roles based on gender and their different contribution to the social system. As it is based on the family as the most integral component of society, the gendered roles perform the social functions of the reproductivity and of the transmission of values - and roles - throught the generations since they served as an effective means of keeping the family system functioning properly (Parsons, 1951; Parsons \& Shils, 1951; Parsons, Bales, \& Shils, 1953). This sociological framework didn't clearly separate the biological and cultural components of the individual identity yet, and it didn't explain what could happen when the social order is smashed by radical changes as a war, a crash or a social revolution (Hawke, 2007): are people satisfied by the gender role they achieved by the birth? If the social changes transform the role differences in gender inequalities and the previous social order in an unbalanced system what new meaning the gender aspects of the individual identity assume? The experiences of the female role as breadwinner during the WW2 and the social transformation after the War proposed critical review about the funcionalist theories and a 'systemic' asymmetry like the wage gap between men and women was described as a result of gender bias and not of choices men and women make about family-work roles (Id.). 
An interpretation as relevant as counterposed can be found among the conflict theorists which interpretation of these gender biases rests on the traditional power unequal distribution in favor of the male roles. According to conflict theory, society is a struggle for dominance among social groups that compete for scarce resources and regarding the zero-sum resource of the power, they represent men as the dominant group and women as the subordinate group. This is traditionally fixed in many of the social structures, which patriarchal order justifies the minor value of the female role in work or in family and their rare public relevance, according to the prevailing rules for success and opportunity in society created by the male dominant group (Farrington \& Chertok, 1993).

As the functionalism, the conflict theories read these dynamics inherent to society just in the pre-industrial era: the Engels' analysis (1884) of the men-women relationship in the family due to the women's dependence on men for the attainment of wages shows that the cultural identification is not far from the sexual aspects, better the biological ones, if we refer to the reproductive function of women and its consequences for thei social roles. Risman and Johnson-Sumerford (1998) confirm this connection also in the contemporary societies with the test of female wage earners that continue to be subordinated in the family, if not for the economical resources, because they must carry most of the domestic duties.

It is just in the recent decades that sociologists - as the other social sciences analysts have tried to observe gender from a different point of view, firstly not connected only with the biological aspects associated and, then, declined in the very different social interactions as the changing societies. The hypothesis can be a gender as the result of interactions among individuals that reveal the gender as a not stable, fluid social structure, not so linked to the biological status and chameleon by default (West \& Zimmerman, 1987). For these interactionist theories a very important function is carried out by the symbols used in the human interactions and their shared current meaning, that is socially created and not natural, but, exactly, fluid, not static. It's an essential point not only for the discontinuity with the past, but also for a not continuity in the presents, especially if we assume that the contemporary societies experiment global meaning and very rapid social changes. So, in the same time gender becomes what we do or perform and not something we are, and its meaning could change quickly, simply moving to a different culture or in a short period. For the interactionism, both gender and sexuality are socially constructed, that is the attribution of their definition through the way people see and experience sexuality sex and gender-linked behavior.

These assumptions arise in a changing cultural condition that favor the belief that men and women are not socially, but only biologically different and that their biological identity could also reverse. The recent scientific and technological acquisitions have sensitized the Western and so-called advanced societies - and their social analysts which awareness enforces the critical position against the persistencies of values, world views, norms, and everyday behaviors that create inequality and injustice based on gender and accuses the traditional structures of the non-Western countries. This is the profile of the feminist theorists that conform the classical conflict theory to the contemporary inequalities in gender-related issues (Hill Collins, 2000). Even if there were very radical cultural and societal changes, in the families as in the workplace the male dominance and the patriarchal values are perpetuating, taking advantage from the powerful message of the media or thanks to the deceiving display of the female 
figures for their exterior and physical features. While specific guarantee norms tend to balance the gender conditions following some rare example of matriarchal system as the Indomensian one (Sanday, 2004), most of the female social and cultural achievements of the last decades seem to be far from their acquisitions and, with them, those of the other vulnerable social actors.

The analytical tools for the interpretation of these phenomena are more and more effective and, in their recent evolution, they have mixed the interactionism approach of the gender as a marker of identity with the critical theories that assume gender as an inequality factor; but, together with gender this analysis stresses the role of other markers as race, class and sexual orientation. For this framework, the social inequalities lie on social, political, and economic structures, such as capitalism, patriarchy, and centralized institutions and on social interactions that outline individual identities through some specific markers - exactly, gender, class, race etc. - and not other, as personality or merit. These markers are used as interconnected axes of definition of the individual identity, the most defined representation we can obtain in comparison with the analysis of a single factor, as for the example of gender (West \& Fenstermaker, 1995). The intersectional approach describes the nature of the individual identity, but also the 'intersectional' process of building of this identity that Acker (2012) classifies in four different dynamics, from the creation of the hierarchies and the recognition of the central gendered institutions, to the social interactions as origin of the shared meaning of gender, to the self- and other identitification based on this meaning.

One of the most relevant aspects of the recent evolution of the analysis of gender as social factor interconnected with the others is the biunivocal relation with the social changes: it reflects, but, at the same time, orients the social changes. The implementation of a strategical approach, the gender sensitive, in the not-sociological analysis and the acquisition of the intersectional methods in the sociological researches allow to stress identity differences and convergencies in a culturally various global world and in a not more biunivocal gender asset - see at the revolutionary social and legal recognition of the third gender (Risman, Froyum, \& Scarborough, 2018).

For the sociological frameworks all these analytical implementations have stimulated a new theorethical and methodological formulation of the investigation about gender and, more generally, the social construction of the individual multiple identities (Einsenstadt, 2000). In research fields as, for example, welfare or the human rights, gender appears as one of the most relevant factors of the identity construction (Cotesta, Cicchelli, \& Nocenzi, 2013), but not the key factor and it makes possible to evaluate how gender exactly affects these processes.

It could be outlined a double evolutive process in the role of gender in the sociological research: firstly, the basic concepts provide not only a separation between biological and cultural aspects, but considering their reversibility, reciprocal influence and the assumption of the sexual dimension; then the processes affected by the gender, together with the other factors, are, more than in the past, but, above all, deeply analysed thanks to detailed and interacting methodological tools (Decataldo \& Ruspini, 2014; Saltzman Chafetz, 2006).

Today, it's more pertinent to describe the sociological research about gender with a specific connection to the gender sensitive approach - that assumes the gender factor as the starting point to explore the individual identity, the process of construction of the 
identities, the relationship with the Other and the common definition of sharing values. It's as much relevant the contribution of the gender oriented approach, which starting points are the social differences and, among them, the gender diversity for the investigation of the social changes, especially with reference to the individual identity lied on four main dimensions: the sex, gender, sexual orientation and gender role (Risman et al., 2018).

The attention for these four components in each stage of the sociological research without their reduction to four variables, allows the overall individual identity, guides the analysis from the research planning, to the theories support, the collection of the second-hand data, the empirical project and the interpretation of the outputs. It's not by chance that some relevant research lines have been recently 'gender oriented': the analysis of the social action and of the values, expectations and behaviors; the ways for an authonomous life project; the access and recognition of the human and social rights; the use and the management of the social resources etc. In the recent sociological handbooks and reports, specific chapters talk about gender in the workplace highlighting different types of mobility and upgrading, occupational segregations, time budgeting, and gendered strategies for the economical growth (Shipman \& Kay, 2009); about embodiment, that is the totality of the ways the cultural ideals of gender creates expectations for and orients the choices about the form of bodies in each society in a different way with a biunivocal relationship between biology and culture (Wolf, 2009); about the globalization as a social process that intensifies the relationships of culture, people and economic activities with a new outline of time and space, of the multiple identitites of each individual and their social roles (Risman et al., 2018); about the third or 'non-binary' gender in a culturally driven reinterpretation of previous not male or female identities like the 'two spirit' in the Native american tribes or the Fa'afafine in Samoa (Kumar, 2017).

These are some of the issues that the recent sociological research have delved into and that a lexicometric analysis can find in an international journal of sociology repertoire for its readers in the last two decades, as the following paragraphs try to verify.

\section{Data and methods}

The analysis of the International Review of Sociology papers was carried out mainly through a lexicometric approach, integrated with a hermeneutical analysis useful in both the first and the last phase of the study.

The first phase has regarded the collection of the corpus, while the last one has concerned the interpretation of the results obtained from quantitative and automatic procedures. The lexicometric analyses, supported by the software IRaMuTeQ, ${ }^{3}$ were carried out to extract the most relevant forms/lemma and to apply some exploratory techniques for identifying the main lexical-textual dimensions, the relationships between some keywords, the recurring topics, and possible differences over the time analysed.

\subsection{The corpus: selection criteria and analysis}

The texts analysed in this study have been collected from the archive of the International Review of Sociology, considering the papers published from 1997 to 2017. 
In the first stage, all the papers which propose the term gender in title were extracted, abstract, body text and/or key words: they were 235, distributed over the past 20 years, as shown in Table 1.

Then, only those papers which had a relevant reference to gender as theoretical or empirical category were selected - and not only as a composing part of a title of some sources, a statistical variable, or synonym - in order to outline meaningful remarks for the aims of each article. This selection has been supported by a hermeneutic analysis, based on careful reading of the abstracts to evaluate the centrality of the gender issues in their hypotheses and theses, as in the implementation of the theoretical and/or empirical methodologies. They resulted 67, distributed over the past 20 years, as shown in Table 1.

The incidence of the selected papers on the extracted ones (SP/EP\%) highlights the increased relevance of the term gender over time: it is used, especially since 2009, more and more often as analytic category in sociological research, rather than as a synonym or to indicate only a demographic characteristic of individuals.

The corpus, submitted to the subsequent analysis, includes therefore 67 selected papers, and has the following lexicometric measurements: dimension $N=495470$, word types $V=$ 21680; Type/token ratio $V / N=4,38 \%$; Hapax $/ V=41,56 \%$; Hapax $/ N=1,82 \%$.

These characteristics show that the corpus can be considered sufficiently large for a quantitative approach analysis (Bolasco, 1999, p. 203).

\subsection{Strategy of analysis}

The analyses on the corpus was carried out with IRaMuTeQ according to the following steps:

(1) Lexical Analysis, accomplished by the exploration of the lexicon used in the corpus, the identification of theme-words/lemma; the lexicalization, ie the individuation of composed textual forms to be considered as single forms;

(2) Analysis of different lexical and semantic dimensions by time and by author/authors gender, carried out through Lexical Correspondence Analysis (LCA), applied to the Aggregated Lessical Tables (ALT) Lemma/Texts (Lerbart \& Salem, 1994). In these tables, the texts were identified respectively according to the different years of publication $(Y=1997,1998, \ldots, 2017$; ) and the gender of the author/authors $(G=1$ Female; 2-Male; 3-Male and Female). The selected lemmas are those 'characteristic' by year or by sex, that is, the lemmas over or underrepresented in a text according to algorithms based on the hypergeometric probabilistic model (Lafon, 1980; Lebart,

Table 1. Extracted and selected papers.

\begin{tabular}{lccc}
\hline & Extracted Papers (EP) & Selected Papers (SP) & SP/EP\% \\
\hline $1997-1999$ & 19 & 2 & 10,53 \\
$2000-2002$ & 18 & 3 & 16,67 \\
$2003-2005$ & 22 & 3 & 13,64 \\
$2006-2008$ & 21 & 3 & 14,29 \\
$2009-2011$ & 45 & 20 & 44,44 \\
$2012-2014$ & 55 & 15 & 27,27 \\
$2015-2017$ & 55 & 21 & 38,18 \\
Total & 235 & 67 & 28,51 \\
\hline
\end{tabular}


Salem, \& Berry, 1998). The analysis of them allows to identify specific features of documents;

(3) Identification of main topics, through descending hierarchical cluster analysis applied to the Lexical Table Text Segments / Lemma (Reinert, 1983). IRaMuTeQ allows the decomposition of the corpus in order to analyze units of basic context or Text Segment (TS), smaller than the complete texts from which they derive that, being scientific articles, are extended (on average about 7300 words) and heterogeneous within them. Instead, the TS are composed of one or more consecutive lines of text of about 40 words which end with a punctuation mark or a space. In this way, it is possible to analyze shorter meaningful local contexts, supposedly more homogeneous than the entire paper. The lexical table TS/lemma has the value 1 when the lemmatized form is present in the TS and the value 0 when it is absent: it is therefore a sparse matrix with binary codes to which a hierarchical descending cluster technique has been applied, suitable for tables with a high number of 0 . The descending classification groups the basic contextual units into classes, or lexical worlds (Reinert, 1993) differentiated by the distribution of their lemmas.

\section{Results}

\subsection{The lexical analysis}

The analysis proposes some basic findings about the lexicon used in the corpus and given text forms with their repeated segments. In particular, the word gender and its repetead segments (see Table 2) are articulated in a composition of sociological concepts and categories that reproduces the variety of research lines we have described in the evolution of the sociological gender studies (Blackburn \& Jarman, 2005) (Par. 1).

Some social conditions as equality and inequalities, stereotypes and segregation have as frequent trends as analytical issues of the gender studies - statistics, mainstreaming, gender sensitive and neutral - highlithing an experienced research on social process and phenomena that has more and more developed a theorethical interpretation about.

The identification of the repeated segments led to the lexicalization of the corpus, aimed at keeping in the following analyses, some complex forms (eg gender_segregation;

Table 2. The Lexical analysis: the word gender and its repeated segments.

\begin{tabular}{lr}
\hline Types & Token \\
\hline gender & 1565 \\
gender_equality & 314 \\
gender_relations & 97 \\
gender_difference & 93 \\
gender_mainstreaming & 44 \\
gender_statistics & 43 \\
gender_role_attitudes & 32 \\
gender_neutral & 28 \\
gender_sensitive & 23 \\
gender_perspective & 20 \\
gender_stereotypes & 18 \\
gender_knowledge & 18 \\
gender_segregation & 17 \\
gender_inequalities & 14 \\
\hline
\end{tabular}


child_care) full of meaning for the objectives of this work, instead of simple forms (e.g. gender and segregation; child and care). ${ }^{4}$

The theme-words, that the treatment with Iramuteq delivers, consist in 17.359 lemmas (only full words, nouns, adjectives, verbs) which trends of occurrencies seem to focus on actors and environments of the social processes analysed - woman, man, mother and child, and, also, family, work or country - that stress the identity and the role of the individual in the social facts. This is one of the most important stage in the sociological research evolution about gender with the recognition of new relevant subjects and dimensions for the social action (Kimmel, 2000) (Table 3).

\subsection{The different semantic dimensions by time $e^{5}$}

The analysis of the specific lexicon, with a particular attention for selected lemma and complex forms by time and by author/authors gender is a focused target for this research to explore the evolution of the investigation lines about gender and the cultural influence in the research objects and the scientific background of the author(s) whose articles are examined.

Taking the time into account, we have resorted to a LAC, carried out considering characteristic lemmatized full words per year, ${ }^{6}$ further selected for frequency threshold $>=20{ }^{7}$ Therefore, the input matrix is an aggregated lexical table (lemmas/years, size $1894 \times 16)$. The first two axes explain $19,49 \%$ of the total inertia $(10,11 \%$ for I axis; $9,38 \%$ for II axis). The lemmas configuration on the first factorial plan suggests that four main different thematic fields (or semantic dimensions) can be identified: they can explain the meaning of the polarity of a first and of a second factor characterized by the lemmas with the higher concentration. (Figure 1)

Table 3. The Lexical analysis: the theme-words.

\begin{tabular}{lr}
\hline Lemma & Token \\
\hline woman & 4271 \\
family & 1905 \\
man & 1885 \\
work & 1725 \\
gender & 1565 \\
child & 1466 \\
social & 1455 \\
country & 962 \\
study & 959 \\
mother & 818 \\
role & 779 \\
time & 764 \\
policy & 728 \\
group & 728 \\
father & 725 \\
society & 717 \\
female & 656 \\
male & 646 \\
position & 624 \\
state & 621 \\
political & 618 \\
model & 617 \\
show & 609 \\
case & 607 \\
parent & 601 \\
care & 597 \\
\hline
\end{tabular}




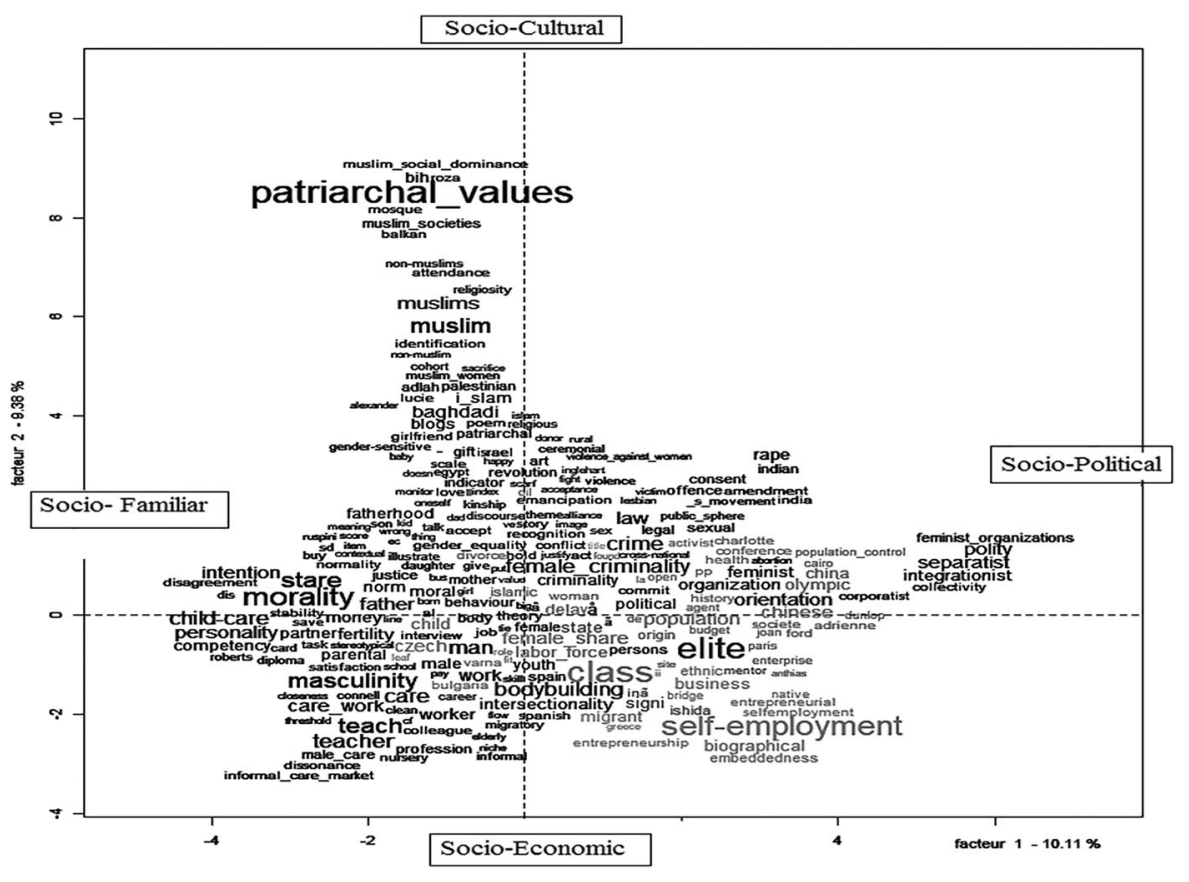

Figure 1. Analysis of the specific lexicon by year: lemmas and semantic dimensions in the first factor plan.

For the first factor, it can be outlined a socio-political field (positive half-axis of the first factor) which the main lemmas are feminist-organization, polity, collectivity, separatist, integrationist, political orientation elite, class; in the opposite pole a sociofamiliar field (negative half-axis of the first factor) is evident with some lemmas prevailing like father, child, child-care parent, mother, fatherhood. Considering the coordinates and the contribute of each year for the first factor, it emerges that if the socio-political themes characterize most of the articles published in 2010 and 1997, the socio-familiar ones characterize most of the articles of the years 2012, 2013, 2015 (Figure 2 and Table 4).

The second factor proposes a lemmas distribution along the axis with a socio-economic field (negative half-axis of the second factor) with lemmas like self-employment, worker, profession, migrant, business, opposite to a socio-cultural field (positive half-axis of the second factor) characterized by lemmas like patriarchal-values, muslim, Bihj, muslim_societies, mosque, muslim_social_dominance. Also, considering the coordinates and the contribute of each year for the second factor, it emerges that the sociocultural themes characterize most of the articles published in the years 2011 and 2016, while the socioeconomic themes characterize most of the articles of the years 2013 and 2003 (Figure 2 and Table 4).

These lexical representations request a more detailed analysis of the local contests where the different lemmas prevail, and some excerpts could be interesting for the conclusive remarks (Par. 4). 


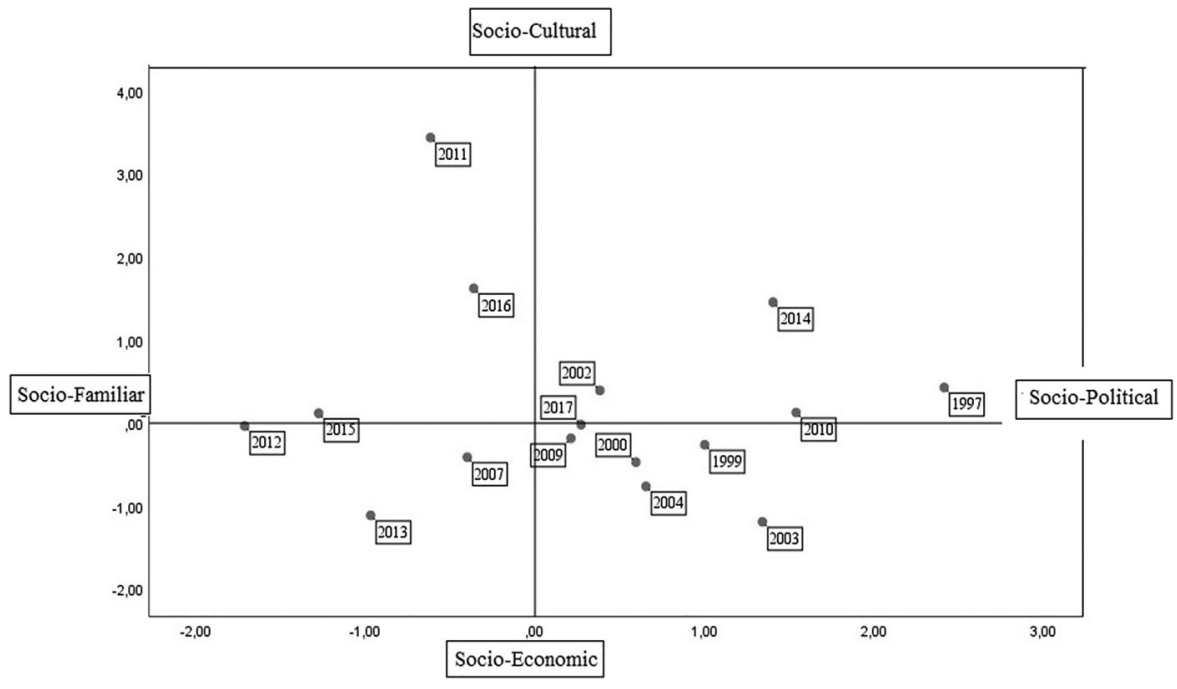

Figure 2. Analysis of the specific lexicon by time: year of edition and semantic dimensions in the first factor plane.

Table 4. Analysis of the specific lexicon by year: coordinates and contributes by factor.

\begin{tabular}{|c|c|c|c|c|}
\hline Years & Coord. factor 1 & CTR factor 1 & Coord. factor 2 & CTR factor 2 \\
\hline 1997 & 2,415 & 0,098 & 0,430 & 0,003 \\
\hline 1999 & 1,002 & 0,022 & $-0,261$ & 0,001 \\
\hline 2000 & 0,596 & 0,011 & $-0,471$ & 0,007 \\
\hline 2002 & 0,383 & 0,003 & 0,394 & 0,003 \\
\hline 2003 & 1,342 & 0,052 & $-1,190$ & 0,041 \\
\hline 2004 & 0,655 & 0,008 & $-0,762$ & 0,011 \\
\hline 2007 & $-0,400$ & 0,007 & $-0,411$ & 0,008 \\
\hline 2009 & 0,212 & 0,005 & $-0,183$ & 0,004 \\
\hline 2010 & 1,541 & 0,298 & 0,128 & 0,002 \\
\hline 2011 & $-0,617$ & 0,013 & 3,438 & 0,394 \\
\hline 2012 & $-1,714$ & 0,164 & $-0,035$ & 0,000 \\
\hline 2013 & $-0,970$ & 0,135 & $-1,112$ & 0,178 \\
\hline 2014 & 1,405 & 0,028 & 1,453 & 0,030 \\
\hline 2015 & $-1,277$ & 0,132 & 0,119 & 0,001 \\
\hline 2016 & $-0,362$ & 0,014 & 1,620 & 0,276 \\
\hline 2017 & 0,272 & 0,010 & $-0,018$ & 0,000 \\
\hline
\end{tabular}

Family migration derives its political significance from the fact that it has become one of the main (and sometimes the only) legal means to find admission in European countries: it is quantitatively by far the largest admission channel for third-country nationals in most western and northern European countries, except the UK, with its large labour migration programme $(2010,20: 1)$

Topics regarding mutual acknowledgement, integration and inclusion of the 'others' (ethnic, gender, physical differences) are becoming hot issues in a multicultural society in which the enlargement of rights and duties to minorities is so relevant to the political agenda $(2009,19: 1)$

In the case of help offered by the employer, the male subjects declined it on economic/pragmatic grounds $(2013,23: 2)$

It seems that women experience expansion as an economic risk (Hakim, 1999) that would not be compatible with the biographical goals of 'autonomy', 'healing', and 'development of the self (2003, 13:1) 
"The autonomization of the vocational aspect of life thus often creates new economic dependencies, which are caused by family interests as well as subjectively understood neglect of family-related socialization commitments" $(2003,13: 1)$

If the thematic analysis suggests some remarks from the gender researches edited by the International Review of Sociology in the different years, the time distribution in the factorial plan of the four fields seems to reveal a not evident precise chronological order in the different declinations of the gender issues: sure enough that the contribution of the articles for year of edition in the four quarters is truly varied (Figure 2).

This temporal variety strenghtens some persistencies in the four selected dimensions during the two decades: for instance, the semantic sociopolitical lemmas in the IRS production propose, from the end of Ninenties to the second decade of the new century, the lasting contest between traditional categories as class, elite, public sphere and a growing use of new categories, rising from new social phenomena. The sociological exploration of the social transformation resorts to solid tools without a fitting work of the cognitive categories at the same time. This is recurring when the sociological analysis is comparative, especially in cultural terms, and the exploration of the social and cultural diversity is done using categories which are patently culturally oriented - e.g. the socio-economic field (negative half-axis of the second factor) and the references to 'patriarchal values'.

\subsection{The different semantic dimensions by author(s) gender}

Then, the Lexical Correspondence Analysis can support the investigation about the gender of the authors of the articled edited that, individual or in team, are $71,2 \%$ female, $19,7 \%$ male and 9,1\% mixed. The analysis outputs consists in two factorial axes from characteristic full words per gender (freq $>=20)($ ALT $1894 \times 3)$ that explain $100 \%$ of the total inertia (61,5\% I axis; 38,5\% II axis).

The lemmas of the articles written by the male authors prevail in the extraction of the first axis, while the lemmas of the articles by 'mixed' authors in the extraction of the second one more. (Figure 3 and Table 5)

In the first positive semiaxis lemmas referred to social and familiar processes that involve men prevail - like morality, bodybuilding, teach, fatherhood, population, foundation, paternal-involvemet, family_planning, gender_role_attitudes, feminised_environment - and they are under the observation of the mixed authors. It's evident a different focus in the opposite quarter where lemmas like woman, work, care-work, self-employment, worker, patriarchal-value, muslim, migration, chinese, employee, professional outnumber: there are the lemmas that female authors use more and that exactly highlight the female roles in family, in the workplace and in some other public spaces.

Hence, the first factorial axis gives back a representation of the gender issues from a gendered point of view: the first one, in the positive pole, outlines the traditional male roles, the opposite pole stresses strictly the female roles, but also in untraditional social spaces, as family is.

The second axis seems to be strongly characterized by the articles edited thanks to the collaboration of female and male authors. The negative semiaxis is determined above all by lemmas referring to socio-political issues like class, revolution, capital, egypt, ishida, egyptian, lithuania, japan, reconciliation, war; in the opposite semiaxis they are prevalent lemmas 


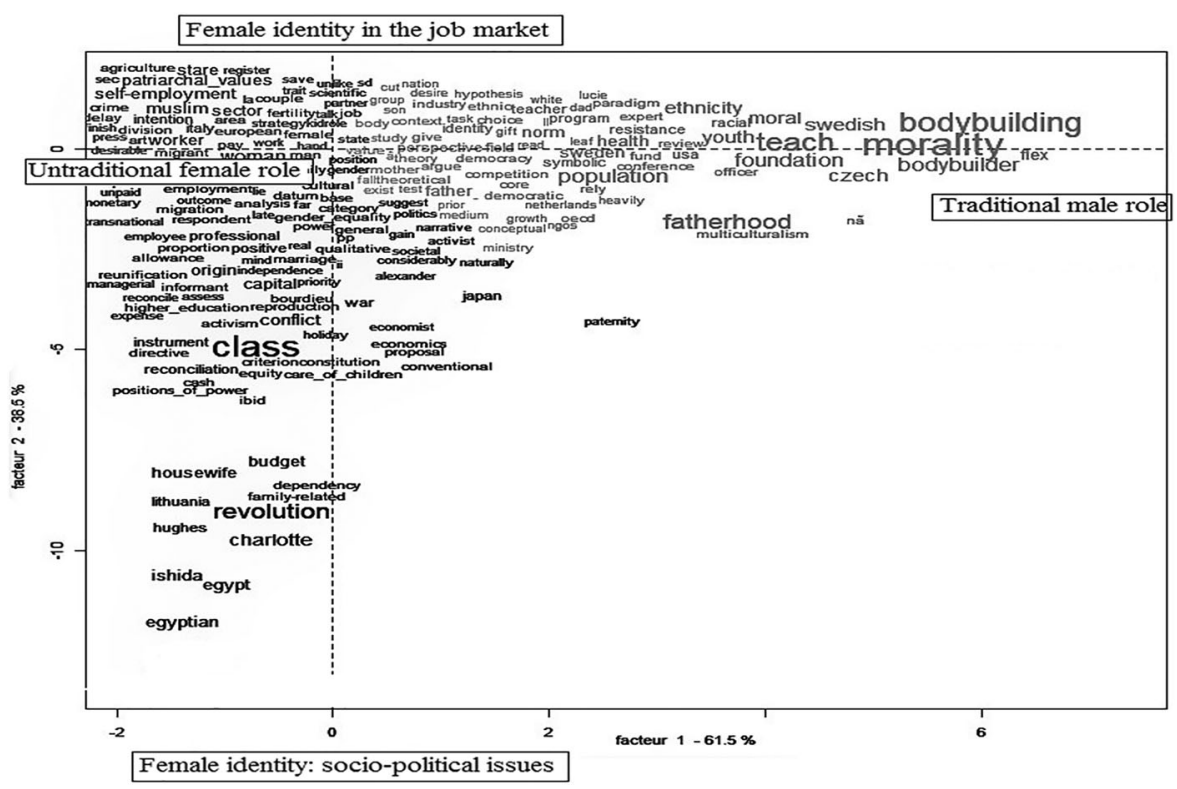

Figure 3. Analysis of the specific lexicon by author(s) gender: lemmas in the factor plane.

Table 5. Analysis of the specific lexicon by author's gender: coordinates and contributes by factor.

\begin{tabular}{lcccc}
\hline Gender & Coord. factor 1 & CTR -factor 1 & Coord. factor 2 & CTR -factor 2 \\
\hline Female & $-0,514$ & 0,189 & 0,364 & 0,095 \\
Male & 1,966 & 0,794 & 0,029 & 0,000 \\
Female and Male & $-0,459$ & 0,017 & $-3,397$ & 0,905 \\
\hline
\end{tabular}

about occupational questions declined in different social conditions like self-employment, female_share, baghdadi, agriculture, female_criminality, competency, i_slam, bulgaria, male_care, counterpublics, care_work, social-worker, work-family. So that, this second axis seems to stress the female social condition in two different settings: a prevalent socio-political area of interest the first and, with reference to the second, to the occupational one, especially underlined the critical aspects of the female identity in the job market.

The following extracts show some of the mentioned lemmas in the corresponding articles, tested the reference content conceived by the authors. The first two are some of the lemmas of the positive half-axis of the first factor used by the male authors and the second two are extracts from articles written by mixed authors in terms of gender:

The gender feminist paradigm enriches 'identity politics' by focusing on 'sexual politics'. Here, explaining how society works and groups interact requires examination of the male heterosexual bias and patriarchal hegemony built into the very structures and practices of social institutions and social discourse $(2009,19: 1)$

Indeed, 'hegemonic masculinity' works through the following simplistic but efficient message regarding the use of the body: the 'real' male is a heterosexual man who is strong, whose strength can be seen through the size of his muscles, and the size of his muscles is the best way for him to appear stronger than other men, as well as to become more attractive to women $(2017,27: 3)$ 
The 1975 SSM, however, surveyed only men, and could tell us nothing about women's relationship to class or about how gender might have affected the presence, the absence or the configuration of class patterns. (2004, 143:2)

Revolutions remain a type of civil struggle which is rather understudied in previous scholarly research. Existing studies on the topic reveal, however, that a revolution's transformative power may facilitate positive changes to women's representation and rights $(2003,13: 1)$

\subsection{The main topics of gender}

The resort to the Cluster Analysis and the identification of main topics through descending hierarchical analysis (Reinert, 1983) applied to the Lexical Table Text Segments / Lemma, permits to outline a selected number of clusters of textual segments and to highlight thematic areas, regardless of the differences by year of publication and by authors' gender, that the previous paragraphs have just put in evidence. Then, the Cluster Analysis outcomes can verify the previous results resorting to a different analytical approach.

The definition of the final clusters was the outcome of consecutive extractions, starting from 15 terminal clusters, in order to establish a hierarchy of the partitions and some stable classes among the different solutions achieved. ${ }^{8}$ One of the first results has been the steadiness of the structure of the dendrograma. Furthermore, in all the extractions carried out considering the lemmas with frequency $>10$, the $99,73 \%$ of text segments has been clusterised.

An analytical exam of the solution of the dendrograma with 15 classes points out a partition in 5 classes: they are clearly defined and two of them could be further broken down in the sixth and seventh clusters. The seven clusters partition allow a more detailed analysis of the meaning contexts without an excessive fragmentation (Table 6).

This solution highlights seven different thematic areas and they can outline the following specific orientations of the gender research in the Review's articles:

- Cluster 7 (23,3\%): family, childcare, regulation of the parents'roles (characterizing lemmas are child, mother, parent, father, leave);

- Cluster 6 (3,9\%): patriarchial values, muslim culture (characterizing lemmas are muslims, patriarchal value, baghdadi);

Table 6. Cluster Analysis: partitions comparison.

\begin{tabular}{|c|c|c|c|c|c|c|c|}
\hline \multicolumn{8}{|c|}{ Partitions } \\
\hline \multicolumn{2}{|c|}{5 clusters } & \multicolumn{2}{|c|}{6 clusters } & \multicolumn{2}{|c|}{7 clusters } & \multicolumn{2}{|c|}{15 clusters } \\
\hline clu & $\%$ segments & clu & $\%$ segments & clu & $\%$ segments & clu & $\%$ segments \\
\hline \multirow[t]{5}{*}{1} & 28,47 & 3 & 28,47 & 1 & 26,77 & 1 & 18,39 \\
\hline & & & & & & 2 & 1,68 \\
\hline & & & & & & 5 & 2,56 \\
\hline & & & & & & 10 & 3,38 \\
\hline & & & & 2 & 1,70 & 6 & 0,45 \\
\hline \multirow[t]{3}{*}{2} & 40,00 & 2 & 14,60 & 3 & 14,60 & 13 & 14,60 \\
\hline & & 1 & 25,40 & 4 & 25,40 & 8 & 19,99 \\
\hline & & & & & & 9 & 5,41 \\
\hline 3 & 4,39 & 4 & 4,39 & 5 & 4,39 & 14 & 4,39 \\
\hline 4 & 3,88 & 5 & 3,88 & 6 & 3,88 & 15 & 3,88 \\
\hline \multirow[t]{4}{*}{5} & 23,26 & 6 & 23,26 & 7 & 23,26 & 3 & 13,07 \\
\hline & & & & & & 4 & 9,20 \\
\hline & & & & & & 6 & 0,45 \\
\hline & & & & & & 7 & 0,53 \\
\hline
\end{tabular}


- Cluster 4 (25,4\%): socioeconomic policies, strategies of equal opportunities (characterizing lemmas are policy woman, country, economic gender-equality)

- Cluster 3 (14,6\%): employment, gender segregation, gender gap (characterizing lemmas are male, female, labor forces, placement);

- Cluster 2 (1,7\%): masculinity, body, bodybuilding (characterizing lemmas are bodybuilding, bodybuilder, hegemonic masculinity)

- Cluster 1 (26,8\%): male and female identities (characterizing lemmas are masculinity, morality, feminist, identity);

- Cluster 5 (4,4\%): family planning, fertility control, demographic rules (characterizing lemmas are population, china, family planning, population control) (Figure 4)

The main topics resulting by the discendent Cluster Analysis seems to be truly oriented by the objects of those articles that the Review edited as their specific case studies - see at the gendered economics of the bodybuilding or the Egyptian Revolution 2011-13 or the young Muslim women of Bengali and Moroccan origin in Italy and the European Islam.

It's evident especially for the cluster 2,5, and 6: their percentages, lower than the other ones, underline their limited influence and the specific thematic definition in comparison with the other clusters.

The three main clusters - 1, 4, 7 - are characterized by lemmas that affect many of the articles analysed and the check by clusters of the thematic areas shows interesting similarities with the analysis, carried out in the previous paragraphs, based on the differences over time and by the authors' gender.

\section{Some remarks about the gender studies in a diachronic analysis}

The lexicometric approach for the analysis of the articles published in the last two decades by the oldest journal of sociology outlines general and specific thematic fields of interest connected to gender and, to a relevant extent, it can highlight the different ways in which gender

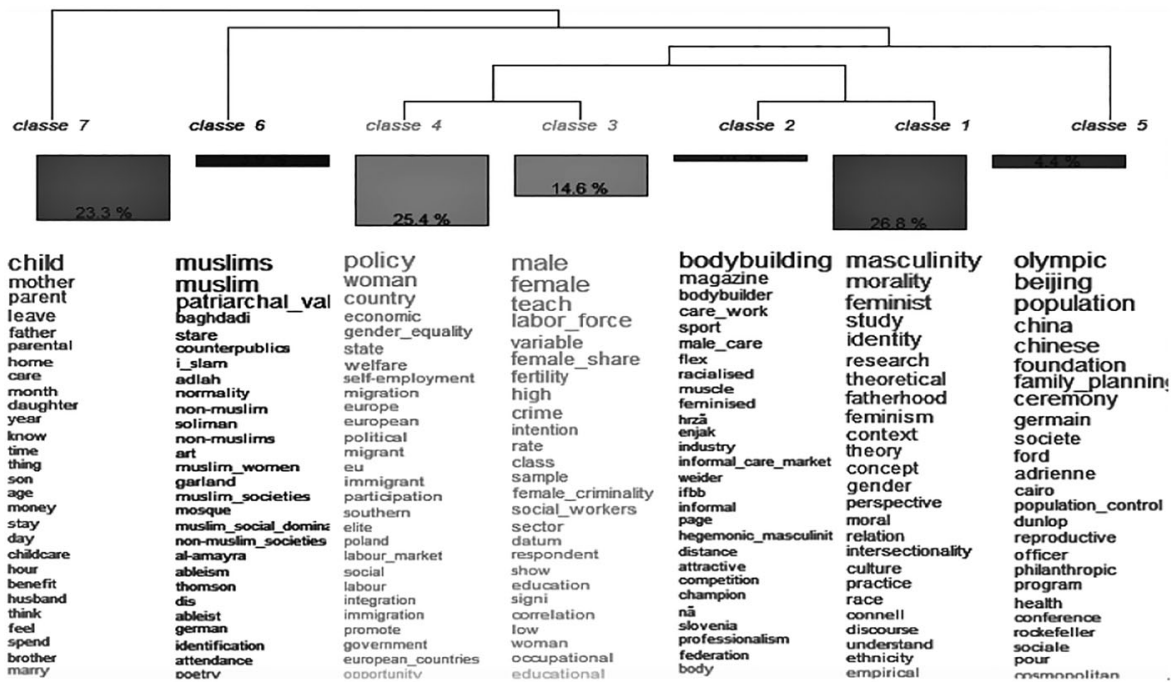

Figure 4. The seven clusters and their main lemmas. 
issues are declined in the international sociological researches presented in the repertoire of the International Review of Sociology. Therefore, it's possible to outline, on both the lexical and the topic level, the changes occurred over this relatively limited time.

The authors are strongly convinced that a sociological journal, even if it's largely known in the international sociological community, only partially represents and, to a lesser extent, influences the gender studies trends in the articles edited in themed sections or research articles and Notes\&Proposals sections.

The International Review of Sociology scientific reputation, its ranking in the upper level of the scientific reviews and its generalist line, however, lure contributions and works that stimulate new ideas, weigh up debates on contemporary sociological thought, or indicate new perspectives in the fields of social theory and empirical research. Furthermore, for the aims and scope of the Review, sociology is not conceived apart from economics, history, demography, anthropology and social psychology, but tries to link the various areas of social activity and to offer up-to-date results of interdisciplinary researches across countries and cultures. Then, even if it's not completely representative, the contributions edited by the Review reflect trends and lines as common as rising for the social sciences.

The thematic outcomes of the lexicometric investigation draft some areas that are essentially verified in all the analysis implemented: they are the macrofields of economy, politics, culture, together with the family and the world of work, as social environments that are 'natural' for gender-oriented research. Despite of this 'natural connotation' of the declination of the gender issues, some evidences stress new research lines: they verify a more mature use of gender as focus point of the sociological work in terms of a richer array of dimensions, subjectivities, cultural expressions: next to traditional concepts connected to socio-political and socio-cultural research areas new ones appear detailing and revising the previous ones (gender-sensitive, fatherhood, kinship etc.).

This improvement is evident also in the higher level of integration of the semantic dimensions that we drafted with frequent areas of overlapping of meaning and common sense related to the prevailing factors. Hence, as the in-depht analysis evolves, the surfacing of complex and varied application of the gender meaning enriches the sociology of gender, especially with reference to its direct links with other specialistic studies and other disciplines researches: for instance, a research on the gender equality requires to join economic, juridical and political categories. This scientific dynamic reflects the social change in relation to the gender identity, the gender process of identification of yourself and the social perception of the gender identity of the Other: the affection of the economic, cultural, political aspects on the biological binary identity empowers the gender factor of the contemporary identities. This explains why some traditional research lines on social processes as those about feminism, labor forces or hegemonic masculinity are replaced by those about equal opportunities, identity and parents' childcare. The institutional management (national and supernational as in the case of the United Nations and European Union) and the public rhetoric (media and social media) build the cultural frame of these social processes.

The Correspondence Analysis first and, then, the Cluster Analysis have inspected the Corpus for specific thematic areas - focused by the articles edited - that are inserted in and across all these macrofields: employment, care, migration, competency, fertility, masculinity, morality. If we can mark common elements among them, in addition to the 
gender analytical factor, they show a crossing connection among the general fields of research and, at the same time, they submit detailed studies from (new) points of view. This connection tests what the lexicometric investigation has highlighted.

The diachronic inspection among the analysed articles did not revealed a gradual evolution for the use of gender by time in the specific research fields. However, the Corpus reveals that current events suggest detailed analysis and growing systematization and specialization in the gender studies that, before, had rapidly focused the most important social areas of investigation (Nocenzi \& Ferrari Occhionero, 2009). So, if an 'evolution' could be drawn, it is integrative in terms of thematic implications explored, methodological tools applied, exchanges and comparisons with other disciplines performed (Healey \& Stepnick, 2017). The integrative modality of detailing the gender research is not only the result of the Review Editorial Board and of the blind referees' evaluation that have allowed the publication of these research articles in the place of others.

The prevalence of specific topics and research lines, reproducing the most popular social facts, reflects also a theoretical and methodological maturation of the sociological research about gender. We have already analysed the inter- and transdisciplinarity and the decomposition in different dimensions. We can confirm that the diversity of the social contexts of analysis allows to explore these different dimensions reinforcing the connection between lexical and topic level.

But, among these new perspectives of the gender research the specificity of the Corpus could join also some evidences about the gender of the authors of the articles. Thanks to the lexicometric analysis is possible to assume that a gendered sociological interest for these issues persists, even if with different trends in compared to the first sociological experiences. The Correspondence Analysis has given back a prevailing focus of the female authors on public action and identity of the gender objects and subjects investigated and, for the male authors, a focus on the individual and private identity. These environments of research seem to explore social contests where the different gender sensitive investigation of the male or female authors aims to delve into emerging and/or evolving issues for the researcher point of view (Kumar, 2017; Risman et al., 2018). Compared to the feminist researches and their activism in a scientific and social way (Hawke, 2007), the authors of the analysed articles investigate on some social processes which frequent common point is the detailed study of a case (e.g. parental leave benefit in Austria, muslin women between secular state and religion) a specific cultural contest (e.g. re-familisation in the Czeck Republic, trafficking women in Mexico) or a comparative analysis (e.g. quality of work and job satisfaction in four European countries or the comparative study on Italian new communities abroad: the cases of Tunisia and South Africa). It seems to confirm that these researchers join the 'traditional' - female - sociologists carrying the research on the pluralization of the classical research dimensions and not, for instance, directly debating on the consolidated categories.

This empirical and phenomenological trend of the gender research arises, at the same time, with and thanks to a growing intersectional integration of the gender factor with the other ones in the traditional thematic areas (political, economical, socio-cultural), but also in new crossing fields of research (e.g. gender identities in the atypical labor markets or in the reproductive choices (Acker, 2012)). As the latest handbooks and reports state, the gender research is more and more enriched by the assumption of its main factor not 
still as the prevalent, but as an integrating variable of a multidimensional investigation: the last step of a long sociological research line that the International Review of Sociology punctually displays.

\section{Notes}

1. This article proposes an extended version of the paper that the Authors proposed at the Conference JADT 2018: $14^{\text {es }}$ Journées internationales d'Analyse statistique des Données Textuelles, Sapienza University of Rome-National Centre for the Researches, Rome, 12th June 2018.

2. See at the International Review of Sociology web site, page 'Aims and scope', https://www. tandfonline.com/action/journalInformation?show=aimsScope\&journalCode=cirs20.

3. IRaMuTeQ is an open software, distributed under license GNU GPL, based on R statistical software and on Python language. It has now reached version 0.7 alpha 2 and it is still under development (Ratinaud, 2009).

4. The lexicalization list includes 110 complex lexicalized entities. Their minimum thresholds of occurrences to consider them in the subsequent analyses are indicated in the following paragraphs.

5. In the analysis by time, it should be pointed out that, as table 1 shows, in the first decade of the considered period the sizes of the sub-corpora are smaller than in the second decade. Furthermore, due to lack of articles, some years in the first decade are not present.

6. The relative frequency of a word in a subtext can be considered as the probability of appearing in $n$ trials (where $n$ is the total number of occurrencies). According to hypergeometric model, the test is carried out under the assumption of a normal distribution, so when the value $z$ is more than $|2|$ we can assume that the word's presence is characteristic $(1-\alpha=95 \%)$.

7. Decreasing the frequency threshold to 10 or raising it to 30 , ACL results were almost stable.

8. To obtain different partitions, the following IRaMuTeQ parameters have been used: Clustering=Simple on text segment; Number of terminal clusters $=5-15$; Minimum frequency of text segment $=5$; Maximum number of analysed forms $=30.000$; Svd method=IRLBA (Augmented, Implicitly Restarted Lanczos Bidiagonalization Algorithm).

\section{Disclosure statement}

No potential conflict of interest was reported by the author(s).

\section{Notes on contributors}

Isabella Mingo is an Associate Professor of Department of Communication and Social Research at Sapienza University of Rome. Her main research areas include measures and indicators of the social processes (free time, cultural participation, social exclusion, information society), statistical analysis of the textual data.

Mariella Nocenzi is an Adjunct Professor of Department of Communication and Social Research at Sapienza University of Rome. Her main research areas include history of the social theory with specific reference to social diversity, social inequalities, gender and sustainability.

\section{ORCID}

Isabella Mingo (D) http://orcid.org/0000-0003-4001-8574

Mariella Nocenzi (D) http://orcid.org/000-0002-2256-4101 


\section{References}

Acker, J. (2012). Gendered organizations and intersectionality: Problems and possibilities. Equality, Diversity and Inclusion: An International Journal, 31(3), 214-224.

Blackburn, R. M., \& Jarman, J. (2005). Gendered segregation and inequality, GeNet Working Paper. Cambridge: Cambridge University Press.

Bolasco, S. (1999). Analisi multidimensionale dei dati: Metodi, strategie e criteri d'interpretazione. Roma: Carocci.

Cotesta, V., Cicchelli, V., \& Nocenzi, M. (Eds.). (2013). Global society, cosmopolitanism and human rights. Newcastle upon Tyne: Cambridge Scholar Publishing.

Decataldo, A., \& Ruspini, E. (2014). La ricerca di genere. Roma: Carocci.

Einsenstadt, S. (2000). Multiple modernities. London: Transaction Pub.

Engels, F. (1884). The origin of the family, private property and the state. Zurich: Hottingen.

Farrington, K., \& Chertok, E. (1993). Social conflict theories of the family. In P. G. Boss, W. J. Doherty, R. LaRossa, W. R. Schumm, \& S. K. Steinmetz (Eds.), Sourcebook of family theories and methods: A contextual approach (pp. 357-384). New York: Plenum.

Hakim, Catherine. (1999). Models of the family, women's role and social policy. European Societies, 1, 33-58. doi:10.1080/14616696.1999.10749924

Hawke, L. A. (2007). Gender roles within American marriage: Are they really changing? ESSAI, 5, 70-74. Article 23.

Healey, J. F., \& Stepnick, A. (2017). Diversity and society: Race, ethnicity, and gender. London: Sage.

Hill Collins, P. (2000). Black feminist thought: Knowledge, consciousness and the politics of empowerment. London: Routledge.

Kimmel, M. S. (2000). The gendered society. New York: Oxford University Press.

Kumar, P. (2017). Radicalizing community development: The changing face of the Queer Movement in Hyderabad City. Community Development Journal, 52(3), 470-487.

Lafon, P. (1980). Sur la variabilité de la fréquence des formes dans un corpus. Mots. Les langages du politique, 1, 127-165.

Lebart, L., Salem, A., \& Berry, L. (1998). Exploring textual data. Switzerland AG: Kluwer-Academic Pub.

Lerbart, L., \& Salem, A. (1994). Statistique textuelle. Paris: Dunod.

Money, J., Hampson, J. G., \& Hampson, J. (1955). An examination of some basic sexual concepts: The evidence of human hermaphroditism. Bulletin of the Johns Hopkins Hospital, 97(4), 301319.

Nocenzi, M., \& Ferrari Occhionero, M. (2009). Gender inequalities: The integrated approach of the gender dimension in Europe. International Review of Sociology, 17(1), 155-170.

Parsons, T. (1951). The social system. New York: Free Press.

Parsons, T., Bales, R. F., \& Shils, E. A. (Eds.). (1953). Working papers in the theory of action. New York: The Free Press.

Parsons, T., \& Shils, E. A. (Eds.). (1951). Toward a general theory of action. Cambridge, Mass.: Harvard University Press.

Ratinaud, P. (2009). IRAMUTEQ: Interface de R pour les Analyses Multidimensionnelles de Textes et de Questionnaires. Retrieved from http://www.iramuteq.org

Reinert, M. (1983). Une méthode de classification descendante hiérarchique: Application à l'analyse lexicale par contexte. Les Cahiers de L'Analyse Des Données, 8, 187-198.

Reinert, M. (1993). Les "mondes lexicaux" et leur 'logique" à travers l'analyse statistique d'un corpus de récits de cauchemars. Langage et Société, 66(1), 5-39.

Risman, B., Froyum, C., \& Scarborough, W. J. (Eds.). (2018). Handbook of the sociology of gender. New York: Springer.

Risman, B. J., \& Johnson-Sumerford, D. (1998). Doing it fairly: A study of postgender marriages. Journal of Marriage and Family, 60(1), 23-40.

Saltzman Chafetz, J. (1978). Masculine, feminine or human?An overview of the sociology of the gender roles. Minneapolis: F. E. Peacock Publishers.

Saltzman Chafetz, J. (2006). Handbook of the sociology of gender. New York: Springer. 
Sanday, P. R. (2004). Women at the center: Life in a modern matriarch. New York: Cornell University Press.

Shipman, C., \& Kay, K. (2009). Womenomics: Write your own rules for success. New York: Harper Collins.

West, C., \& Fenstermaker, S. (1995). Doing difference. Gender and Society, 9, 8-37.

West, C., \& Zimmerman, D. H. (1987). Doing gender. Gender and Society, 1(2), 125-151.

Wolf, N. (2009). The beauty myth: How images of beauty are used against women. New York: Harper\&Collins. 